\title{
Statistical study of ELF/VLF emissions recorded by a low-altitude satellite during seismic events
}

\author{
Michel Parrot \\ Laboratoire de Physique et Chimie de l'Environnement, Centre National de la Recherche Scientifique \\ Orléans, France
}

\begin{abstract}
This paper presents a statistical study of low-altitude satellite data linked to seismic events. Data of the satellite AUREOL 3 are considered during 24-hour windows around the time of 325 earthquakes with $M_{s}>5$. Amplitudes at the output of different frequency filters in the ELF/VLF range, connected to electric or magnetic components, were stored in a file. This file is organized as functions of three parameters: $\Delta t$, the difference between the time of the earthquake and the time of the satellite data; $\Delta \mathcal{L}$ ong, the difference between the longitude of the earthquake and the longitude of the satellite; and $\Delta$ Inv.Lat, the difference between the invariant latitude of the earthquake and the invariant latitude of the satellite. When all data are averaged over time, the amplitude is maximum in the interval $\Delta$ Long $<10^{\circ}$ regardless of $\Delta$ Inv.Lat. This indicates that, due to propagation, waves related to seismic processes can be observed all along the magnetic meridian passing over the epicenter of an earthquake. However, as the AUREOL 3 data were not continuously recorded, thus providing a limited number of events, it was impossible to carry out a complete statistical study.
\end{abstract}

\section{Introduction}

Electromagnetic emissions in the ELF/VLF range that are related to seismic or volcanic activity are well known. Since the reports by Gokhberg et al. [1982] and Warwick et al. [1982], many papers have presented ground observations of wave emissions during seismic events. The more recent work is by Fraser-Smith et al. [1990], Fujinawa and Takahashi [1990], Dea et al. [1993], and Koons and Roeder [1993]. Many other references can be found in the review paper by Parrot et al. [1993]. Two types of emissions can be considered. First, precursor emissions occur a few hours before earthquakes, in a largefrequency range from one hundredth hertz up to several megahertz. Second, emissions observed after the shock generally are attributed to the propagation of acoustic-gravity waves. However, all hypotheses concerning the generation mechanism of precursor emissions are also valid after the shock, when the Earth's crust retums to an equilibrium state. These hypotheses are described by Parrot et al. [1993] and are mainly related to: wave production by compression of rocks [Cress et al., 1987], diffusion of water in the epicentral area [Draganov et al., 1991], and redistribution of electric charges [Lockner et al., 1983; Enomoto and Hashimoto, 1990].

The seismo-electromagnetic emissions can propagate up to the ionosphere [Gokhberg et al., 1983, 1984; Molchanov, 1991], and observations made with low-altitude satellites have shown increases of ELF/VLF waves above seismic regions [Larkina et al., 1983, 1989; Parrot and Mogilevsky, 1989; Chmyrev et al., 1989; Mikhaylova et al., 1991; Molchanov et al., 1993]. Studying the dispersion of whistlers, Hayakawa et al. [1993] have shown that seismic activity can affect the propagation of magnetospheric waves.

Most reports present observations of a single event, and no direct relationship between earthquake and wave growth can be

Copyright 1994 by the American Geophysical Union.

Paper number 94JA02072.

0148-0227/94/94JA-02072\$05.00 seen, although several hypotheses about the generation mechanism exist. Only a statistical study can show the general behavior of wave emissions. Such study is possible with ground observations made close to the epicenters, but it takes a long time to register many events. In contrast to ground experiments, satellite experiments cover most seismic zones of the Earth, and statistical studies become meaningful because of the much larger number of recorded events. Such statistical work was carried out by Henderson et al. [1993], using data from the low-altitude satellite DE 2, but this work indicates no evident correlation between seismic activity and wave emissions. Molchanov et al. [1993], however, have observed electromegnetic emissions associated with many earthquakes when they studied the Intercosmos 24 satellite data. The aim of the present paper is to present another statistical study with data from the low-altitude satellite AUREOL 3. The working method is slightly different as it is not based on values exceeding thresholds, as in the paper by Henderson et al. [1993], but on average values calculated when all events are considered. There is no comparison between earthquake orbits and control orbits, but there is comparison between average amplitudes of waves recorded close to earthquakes and those recorded far from earthquakes. This study also considered the difference between earthquake and satellite longitudes to check if there is a longitudinal effect. Larkina et al. [1983, 1989] found that emissions related to earthquakes could be observed at more than $100^{\circ}$ in longitude from epicenters.

The experiment and data processing are described in section 2. Section 3 presents the results, and conclusions are given in section 4.

\section{The Data}

The ARCAD 3 wave experiment was placed into a quasi-polar orbit onboard the triaxial-stabilized AUREOL 3 satellite (apogee $2012 \mathrm{~km}$, perigee $408 \mathrm{~km}$, inclination $82^{\circ} 5^{\prime}$, period $109.5 \mathrm{~min}$ ), launched on September 21, 1981. ARCAD 3 measured three magnetic and two electric components of the waves in the ELF and VLF range, from $10 \mathrm{~Hz}$ up to $15 \mathrm{kHz}$. The $E_{\mathrm{z}}$ component was approximately parallel to the Earth's magnetic field at high 
latitude, the $E_{H}$ component being nearly perpendicular. The $x$ direction was nearly parallel to the satellite trajectory. All characteristics of the experiment are given by Berthelier et al. [1982]. Two telemetry systems were available on AUREOL 3: the real-time French telemetry (TMF), only used when the satellite was near a telemetry station, and the onboard tape-recorded Soviet telemetry (TMS).

Because of the problem of correlating satellite orbit, earthquake time, and satellite data, only the onboard recorded telemetry has been used. This telemetry contains filterbank data with a frequency range between $10 \mathrm{~Hz}$ and $15 \mathrm{kHz}$. At a given time the filters are connected to two channels among the two electric and the three magnetic components. Data from October 1981 until December 1983 were used without any selection concerning geomagnetic activity. Along the orbit, the recording period was not continuous and varied from one day to another (there are days without any data), depending on experiment programming. Only two different TMS modes were considered: ZAP3 and ZAP4, whose filter sampling frequencies were, respectively, 400 points and 50 points every $128 \mathrm{~s}$. The calibration sequences were removed from the data. A data file was created with all samples recorded \pm 24 hours around the time of an earthquake. Data between $-45^{\circ}$ and $+45^{\circ}$ of invariant latitude were selected to avoid zones of intense natural noise. Data processing of low-altitude satellites have shown that, in this region, the natural noise at frequencies lower then $800 \mathrm{~Hz}$ is generally not intense [Parrot, 1990]. The earthquakes were chosen from the Preliminary determination of epicenters [1981 - 1983], with magnitudes larger than 5.0 and with epicenters located between $-45^{\circ}$ and $+45^{\circ}$ of invariant latitude. For the two years of the mission, the total number of earthquakes with corresponding satellite dats is 325 . The positions of all epicenters are shown on Figure 1. As can be seen, some of the earthquakes occurred below the see, which is certainly not a good condition to observe electromagnetic waves [Oike and Ogawa, 1986], but in the Pacific ocean the many small islands can act as antennae and propagate acoustic-gravity waves.

The data set is mainly organized as functions of three parameters: $\Delta t$, the difference between the time of the earthquake and the time of the satellite data; $\Delta$ Long, the absolute difference between the longitude of the earthquake and the longitude of the satellite; and $\Delta I n v . L a t$, the absolute difference between the invariant latitude of the earthquake (taken at $150 \mathrm{~km}$ above the epicenter) and the invariant latitude of the satellite. The other parameters are the five electromagnetic components because they were not recorded simultaneously, and the filter frequencies of $140,450,800,4500$, and $15,000 \mathrm{~Hz}$. Concerning this data set, two points must be underlined: the same data may refer to different earthquakes if they occurred in the same \pm 24 hour window, but it is equally possible that several data records, recorded at different places around the Earth, might refer to a single earthquake. Several case studies corresponding to this date set can be found in the works by Parrot and Mogilevsky [1989] and Parrot et al. [1993].

\section{The Results}

For each electromagnetic component and each filter frequency, two types of plots are presented. First, the average value of the field is shown as functions of $\Delta I n v$.Lat and of $\Delta L$ Lng with bins of

\section{Epicenters of the earthquakes}

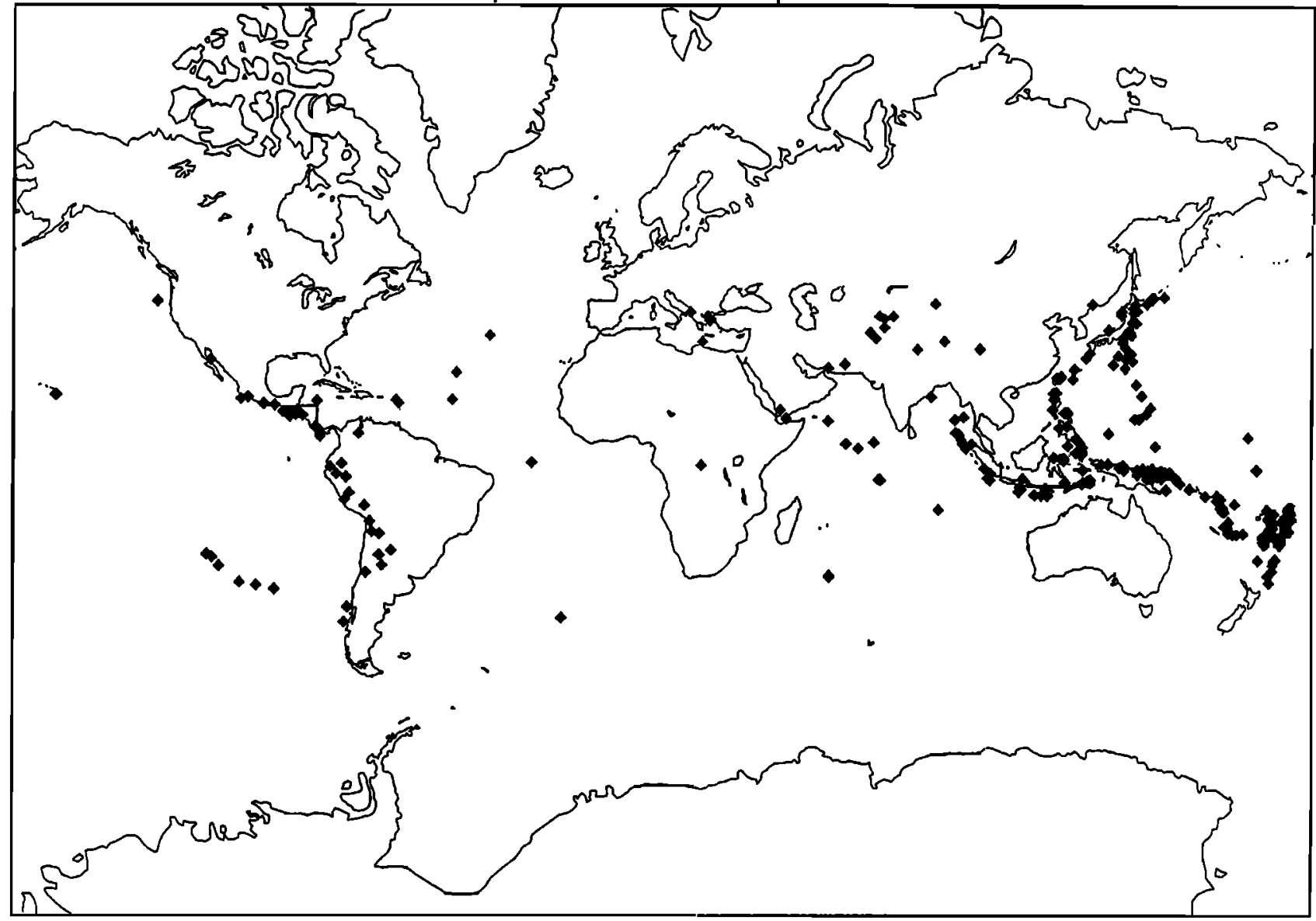

Figure 1. Locations of earthquake epicenters used in the statistical study. 
$10^{\circ}$ or $20^{\circ}$. The data have been averaged over the entire \pm 24 hours around the time of earthquakes. Considering that the waves could easily propagate from one hemisphere to the other, the data in opposite hemispheres are mixed for a given $\Delta L$ Long value. A maximum around the point $\left(0^{\circ}, 0^{\circ}\right)$ is expected if there is a seismo-electromagnetic effect. Second, in order to detect precursor effects, the average value of the field is shown as functions of $\Delta t$ with bins of 4 hours and of $\Delta$ Long with bins of $20^{\circ}$. In this last case the data have been averaged over all invariant latitudes. For each plot the lower panel shows a table with the number of earthquakes (lower number), and the number of data samples (upper number) that were taken into account in all cells.

Plate 1 shows the average amplitude of the signal recorded by magnetic component $B_{X 45}$ at $140 \mathrm{~Hz}$ as a function of $\Delta I n v$.Lat and $\Delta$ Long. All data contained in the set are plotted on this figure: the data with $\Delta$ Long between $0^{\circ}$ and $20^{\circ}$ are relevant to satellite observations close to epicenters, but data with $\Delta$ Long between $160^{\circ}$ and $180^{\circ}$ are related to satellite observations when

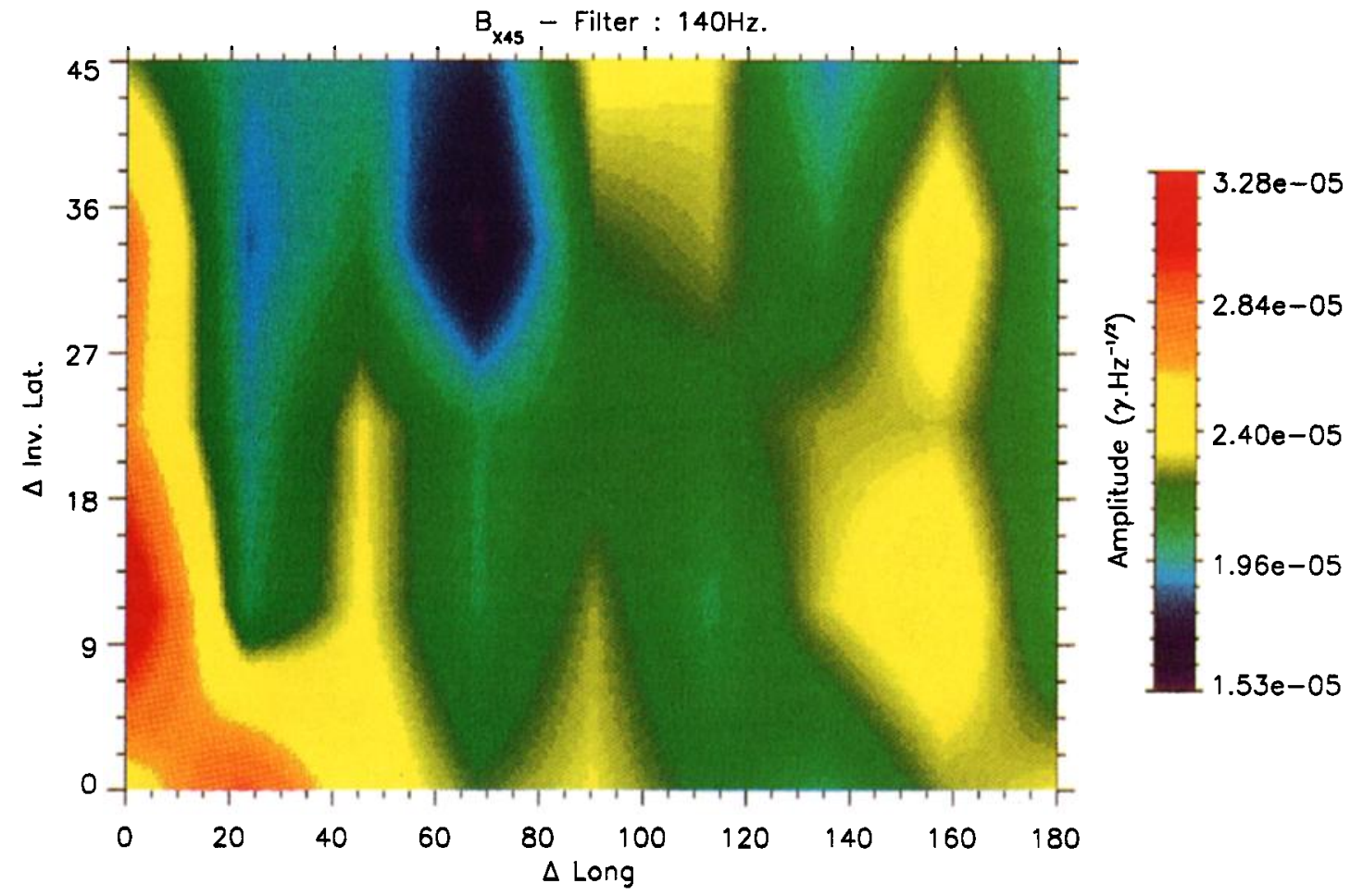

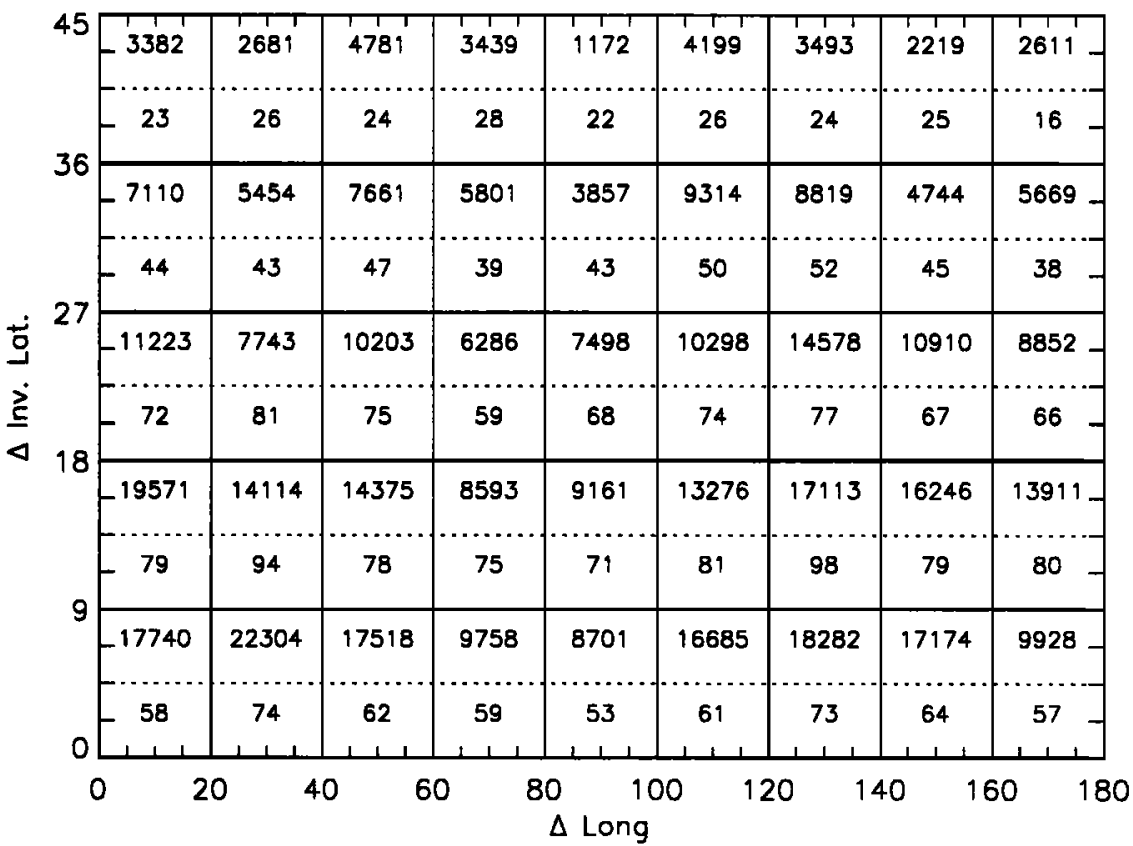

Plate 1. In the upper panel, average amplitude of the waves at $140 \mathrm{~Hz}$ recorded by the magnetic component $B_{x 45}$ as a function of the relative position between epicenters and satellite: $\Delta I n v$.Lat and $\Delta L$ Lng. This amplitude is colorcoded according to the scale on the right. In the lower panel, each cell indicates at the top the number of samples and at the bottom the number of earthquakes, which were taken into account for the upper panel. 
earthquake epicenters were located at exactly the opposite place. An increase of the wave intensity is observed for $\Delta$ Long between $0^{\circ}$ and $20^{\circ}$. This increase is not only present for small $\Delta$ Inv.Lat, but also for larger values. It indicates that, when an earthquake occurs, there is a significant increase of waves in all of the magnetic shell passing through the longitude of the epicenter. As the data were averaged over the \pm 24 hours around the time of earthquakes, we do not know if this emission is a precursor effect or not. Looking to the color scale on the right, it must be noted that there is only a factor of 2 between the intensity level of emissions correlated with earthquakes and the background noise level.
Data in Plate 2 show part of the data shown on Plate 1, now only considering telemetry in the ZAP3 mode. The plot is with bins of $10^{\circ}$ in $\Delta$ Long, and is now limited to $\Delta$ Long $<90^{\circ}$. The lower panel shows that the number of events is slightly different. The increase is also observed when satellite longitude is close to that of the epicenters. No longitudinal effect can be observed in Plates 1 and 2.

The variation as a function of frequency is shown in Plate 3, where the average amplitude of the signal recorded by magnetic component $B_{X 45}$ is plotted. It is the same as Plate 2 (same number of samples and same number of earthquakes), but for three different frequencies: $450 \mathrm{~Hz}$ in the lower panel, $800 \mathrm{~Hz}$ in the

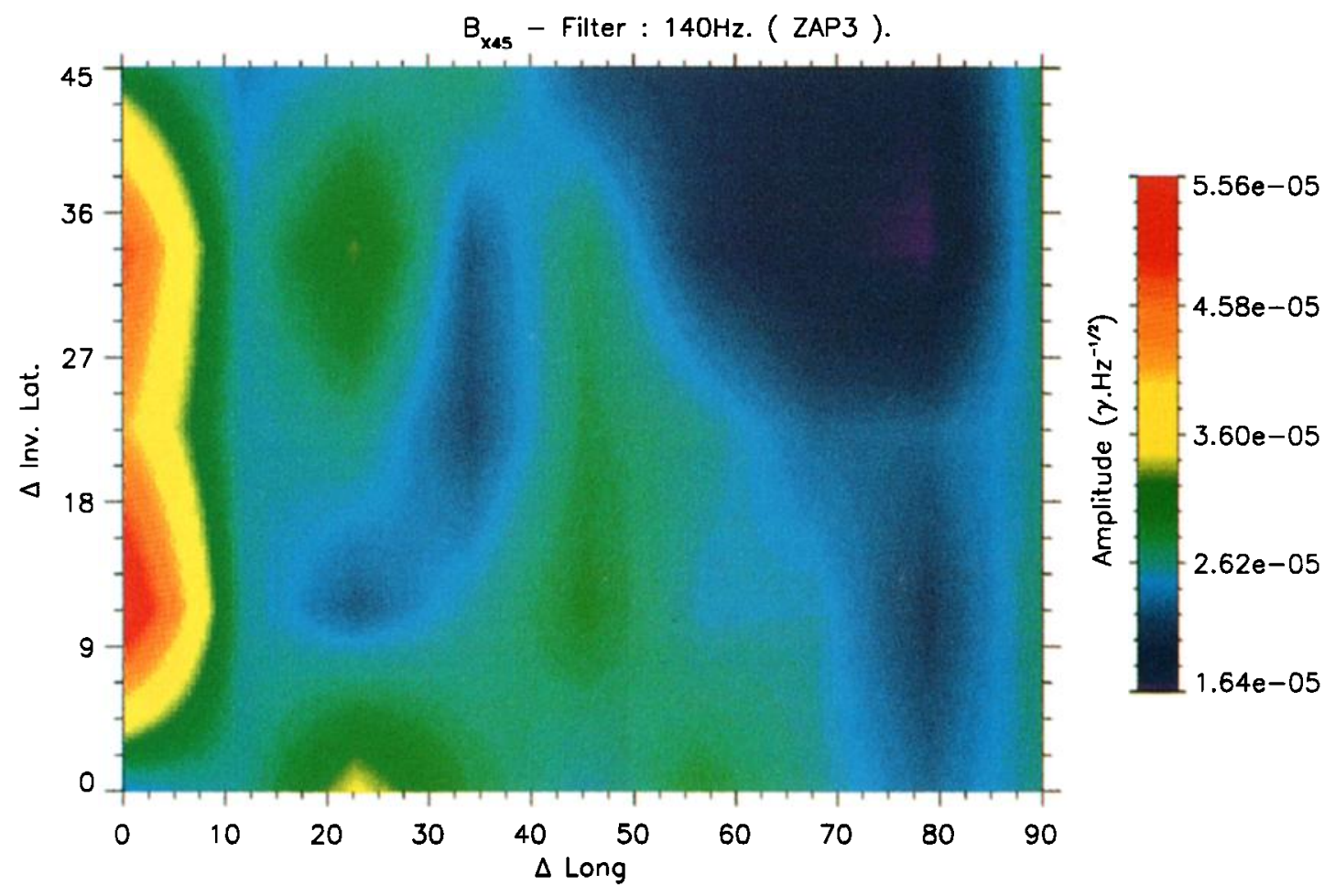

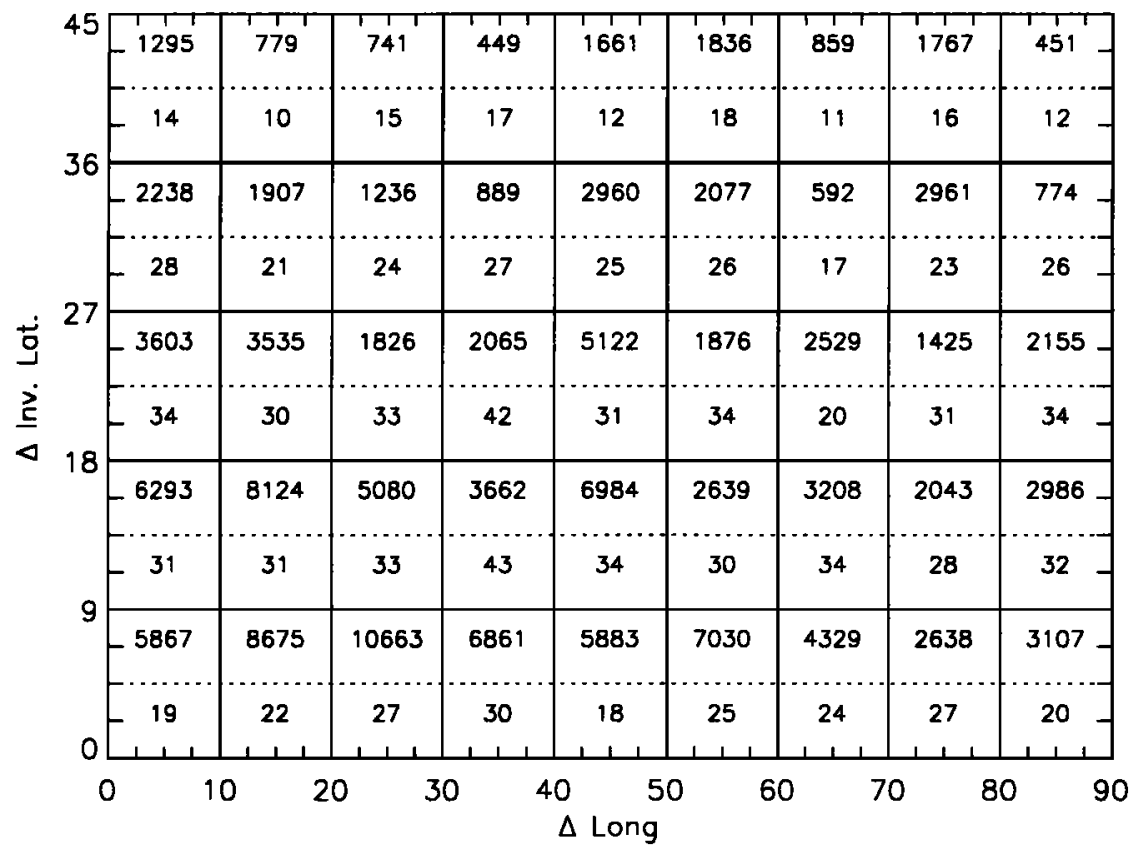

Plate 2. Same as in Plate 1 , but with $\triangle$ Long $<90^{\circ}$ and telemetry limited to ZAP3 mode. 
$B_{x 45}-$ Filter : $4500 \mathrm{~Hz} .($ ZAP3 ).

23,343

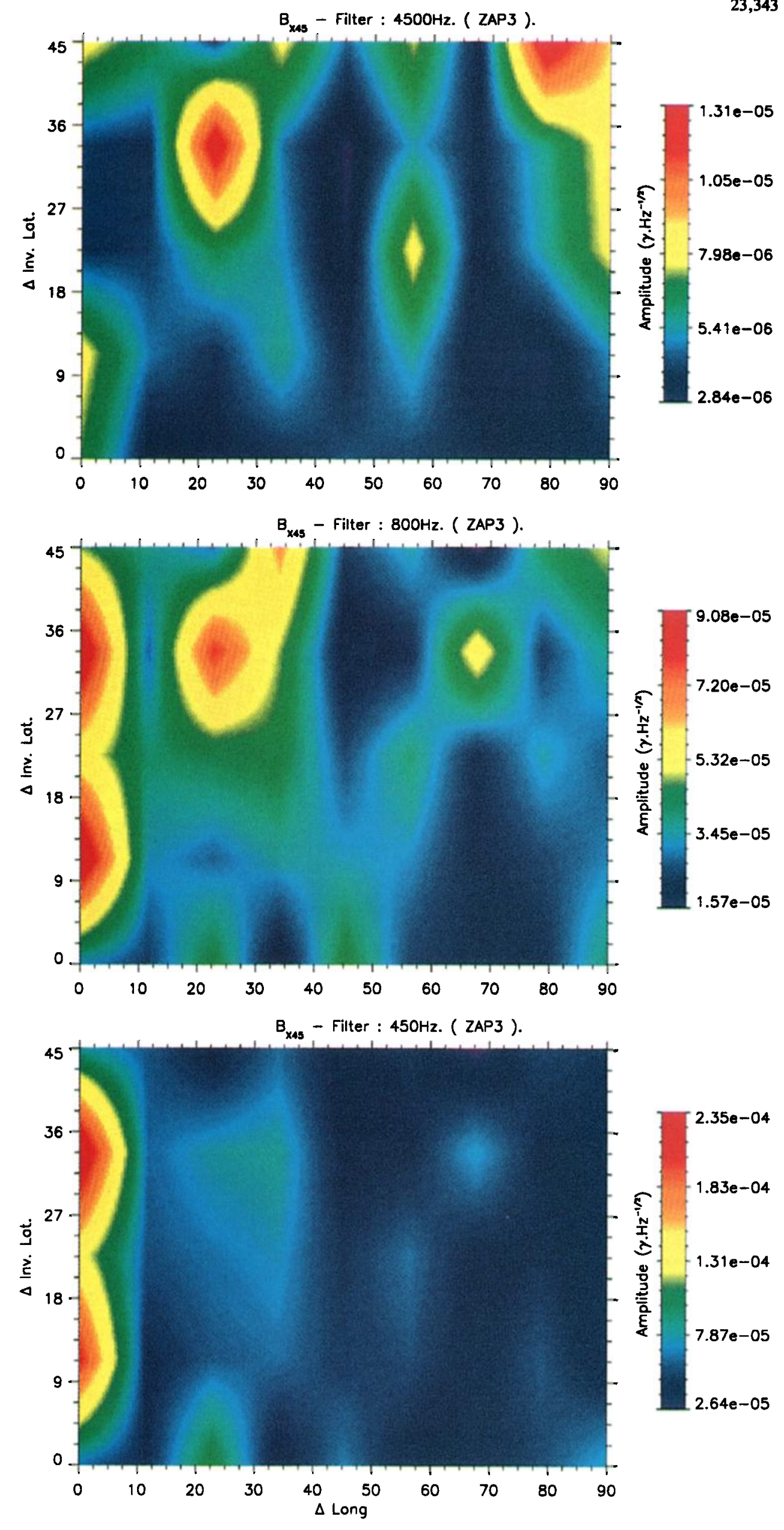

$B_{x A 5}$ - Filter : 800Hz. ( ZAP3 ).

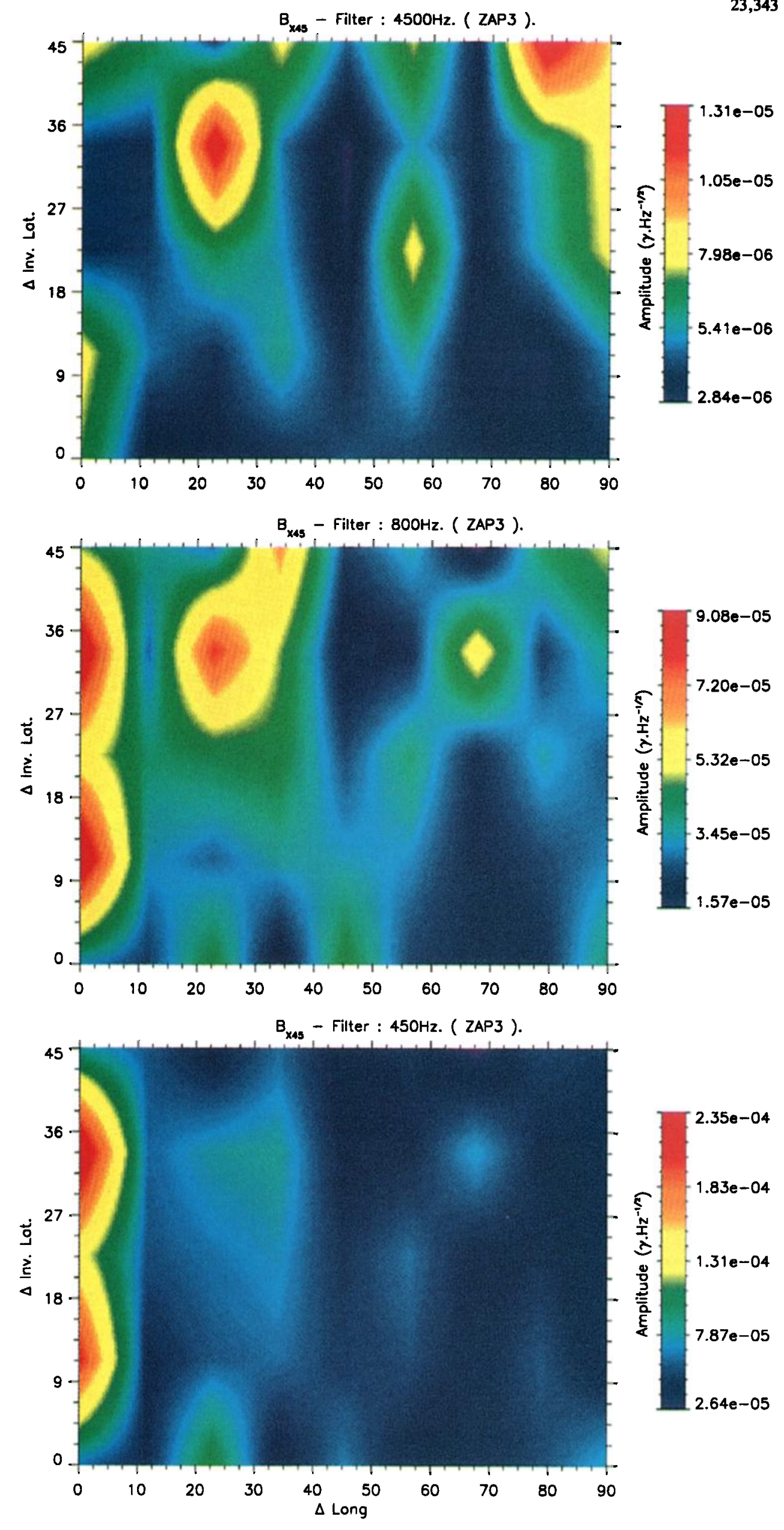

$B_{x 45}$ - Filter : 450Hz. ( ZAP3).

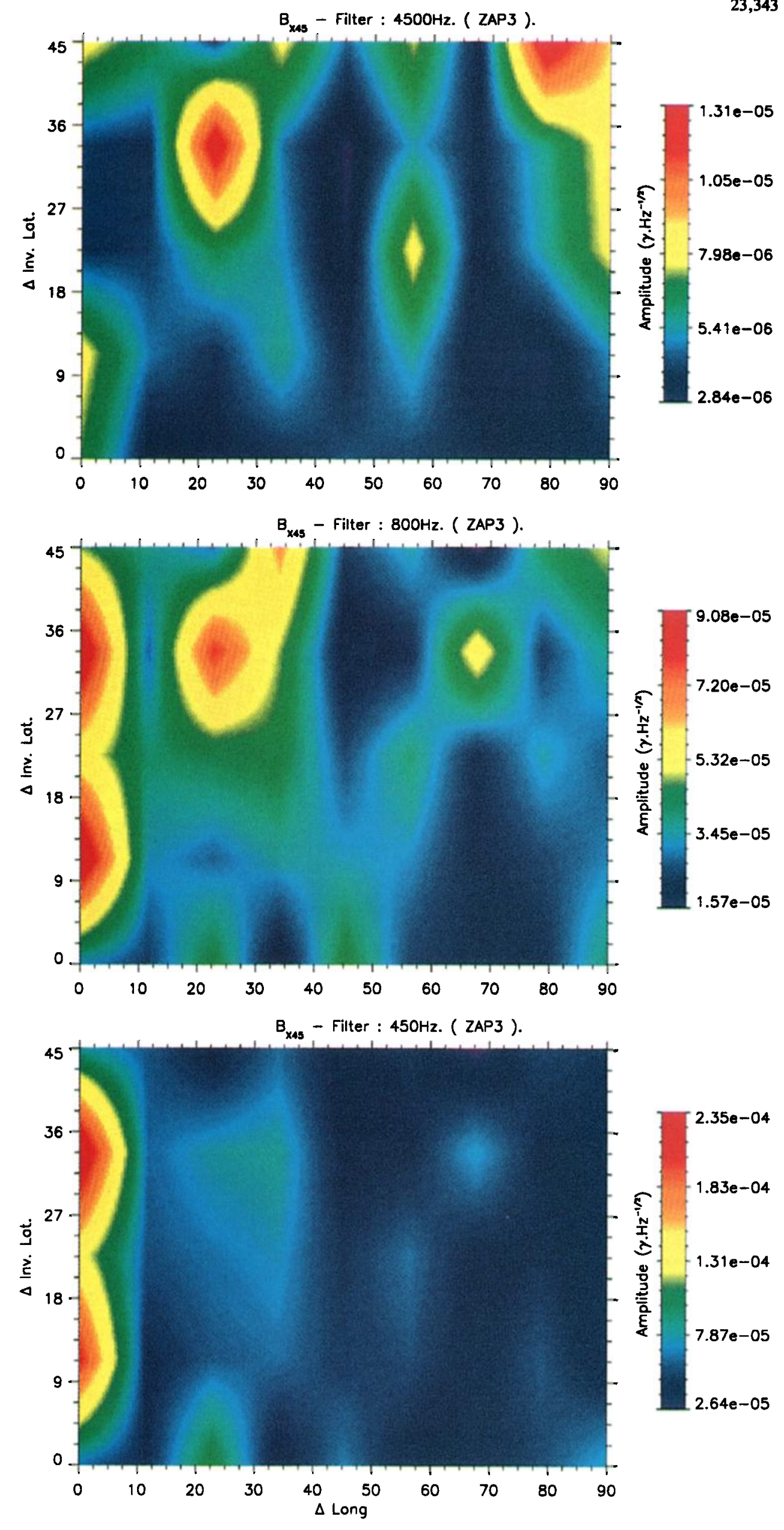

Plate 3. Same as the upper panel of Plate 2, but for different frequencies: $450 \mathrm{~Hz}$ in the lower panel, $800 \mathrm{~Hz}$ in the middle panel, and $4500 \mathrm{~Hz}$ in the upper panel. 

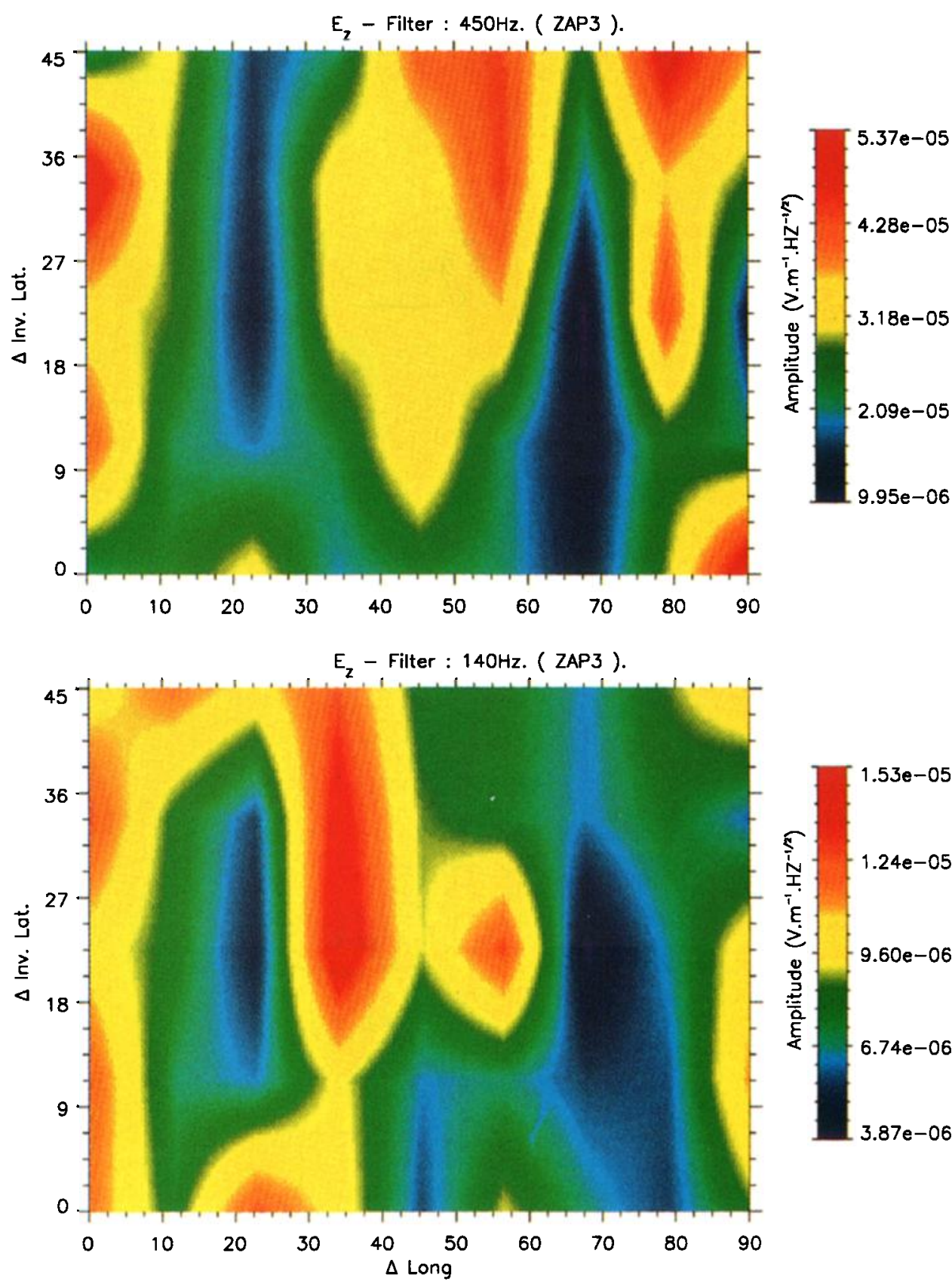

Plate 4. Same as in Plate 2, but for the $E_{2}$ component; the data corresponding to the 450 - and 140- $\mathrm{Hz}$ filters are respectively in the upper and middle panels. The lower panel presents the numbers of samples and events, as in Plate 1.

\begin{tabular}{|c|c|c|c|c|c|c|c|c|c|}
\hline 45 & -1768 & ' 831 & 2111 & 965 & $\begin{array}{l}111 \\
3372\end{array}$ & 2569 & 1260 & 3531 & 2483 \\
\hline & -13 & 16 & 19 & 17 & 18 & 20 & 15 & 23 & 17 \\
\hline 36 & 2560 & 2273 & 1924 & 1596 & 4719 & 3898 & 3506 & 3882 & 4285 \\
\hline & -23 & 26 & 28 & 25 & 33 & 29 & 24 & 29 & 26 \\
\hline 27 & 5198 & 5495 & 2624 & 4080 & 9314 & 5907 & 4337 & 7330 & 3497 \\
\hline & -37 & 40 & 45 & 51 & 49 & 41 & 36 & 47 & 39 \\
\hline & -8791 & 13072 & 4905 & 5242 & 7391 & 7449 & 4918 & 11671 & 5413 \\
\hline & -41 & 43 & 53 & 49 & 48 & 40 & 44 & 50 & 51 \\
\hline$y$ & -9910 & 17714 & 10301 & 9108 & 10914 & 6695 & 6240 & 9124 & 8702 \\
\hline & -31 & 40 & 38 & 42 & 38 & 37 & 38 & 41 & 39 \\
\hline & 0 & & & & $\begin{array}{l}0 \\
\Delta \text { Long }\end{array}$ & & & & 90 \\
\hline
\end{tabular}


middle panel, and $4500 \mathrm{~Hz}$ in the upper panel. We see that the increase in wave intensity close to the epicenter longitude is still strong for the $450-\mathrm{Hz}$ and $800-\mathrm{Hz}$ filters, but that this effect completely disappears at the higher frequency.

One data set for electric components is shown on Plate 4. It concerns the $E_{Z}$ component at $450 \mathrm{~Hz}$ (upper panel), and $140 \mathrm{~Hz}$ (middle panel). The lower panel shows the number of samples and events as in Plates 1 and 2 . As with the magnetic components, an increase is observed when $\Delta \mathrm{L}$ ong $<10^{\circ}$, but other peaks are also seen at different places. This is due to the specificity of electric measurements. In this low-latitude region, Kelley and Mozer [1972] and Holtet et al. [1977] have shown with low-altitude satellite measurements that electrostatic turbulence is very commonly observed around the magnetic equator. This phenomenon mainly occurs during the night. In Plate 4 (and in the others), no distinction is made between the emissions recorded during day or night.

An important point is to known if our statistic can be corrupted by other natural noises like whistlers due to thunderstorm activity near the equator. Numerous whistlers may be occasionally

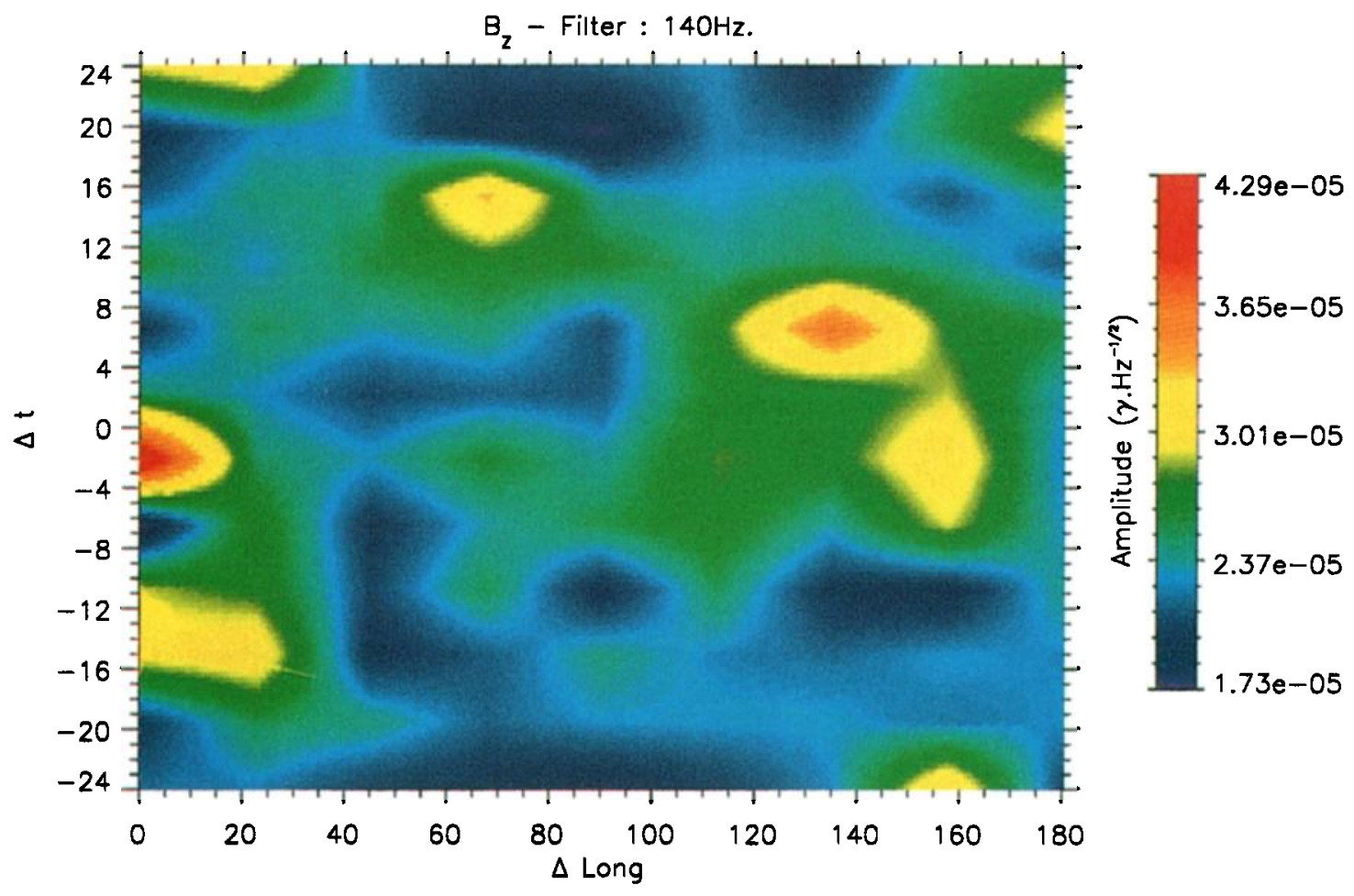

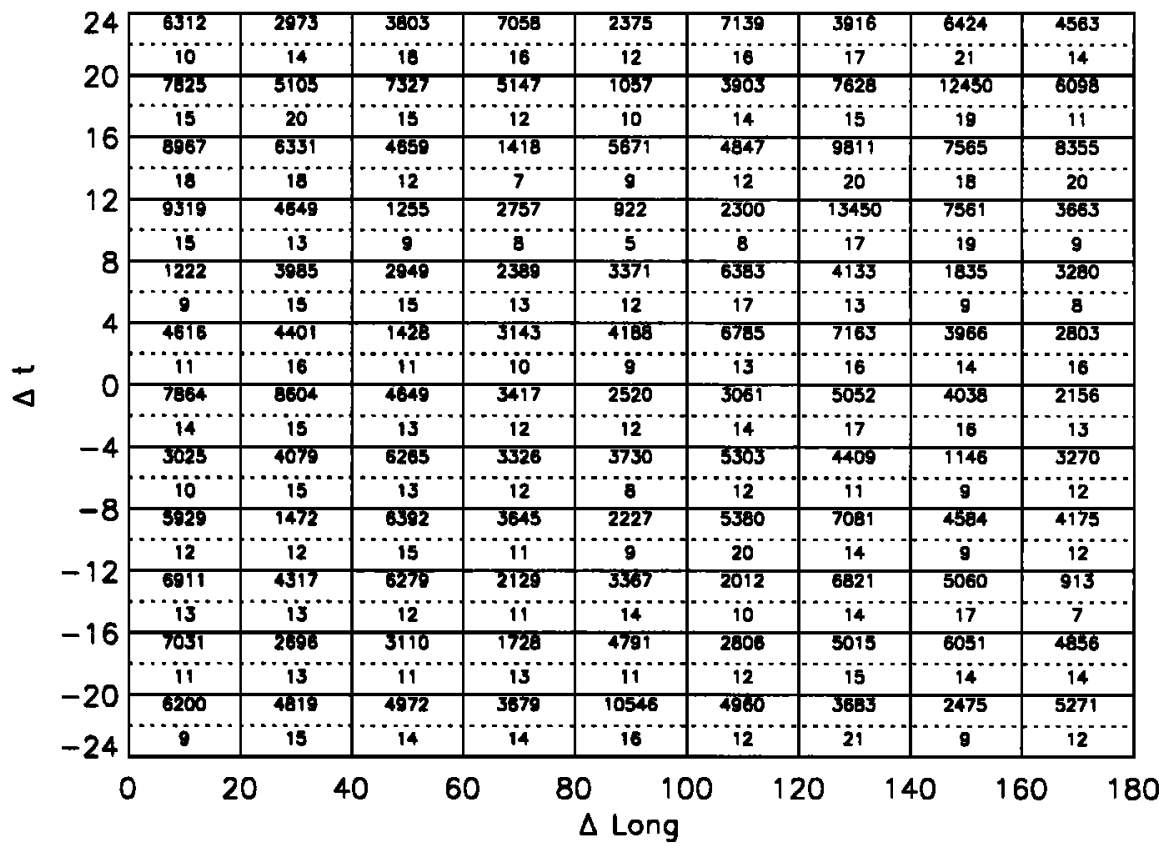

Plate 5. The upper panel shows the average wave amplitude at $140 \mathrm{~Hz}$ recorded by the magnetic component $B_{\mathrm{Z}}$ as a function of $\Delta t$ and $\Delta$ Long. This amplitude is color-coded according to the scale on the right. The lower panel shows the same type of information as in Plate 1. 
observed at low latitudes [Scarabucci, 1970], but their importance is relative. Our data are obtained with filters that measure the mean power of the signal with a logarithmically compressed output and a time delay of $0.3 \mathrm{~s}$ [Berthelier et al., 1982]. Therefore the amplitude of pulses like whistlers are strongly attenuated. In fact, a representation of the data of the low-altitude satellite AUREOL 3 at frequencies around $800 \mathrm{~Hz}$ and in geographical coordinates [Parrot, 1990] does not indicate a relation with regions of high thunderstorm activity (this is not the case at higher frequencies). The ELF background noise level is not very intense at low latitudes and this can be also observed with other low-altitude satellites [Kelley and Mozer, 1972; Kelley et al., 1975; Holtet et al., 1977]. A comparison between particular power spectra obtained from orbits of AUREOL 3 near epicenters and the average power spectrum recorded by AUREOL 3 at low magnetic latitudes is shown in the work by Serebryakova et al. [1992]. Another point concerns the attenuation of waves propagating from the ground up to the ionosphere near the equator. However, until now, we cannot say that the observed wave enhancement is directly related to electromagnetic waves produced by earthquakes at the ground level. Many other hypotheses can be considered [Parrot et al., 1993].

To determine if the emissions observed close to the epicenter longitudes occurred before or after the earthquakes, the complete data set corresponding to magnetic component $B_{\mathrm{z}}$ at frequency $140 \mathrm{~Hz}$ is shown in Plate 5. The average amplitude is now plotted as a function of $\Delta t$ and $\Delta$ Long in the upper panel. The lower panel indicates the number of samples and the number of events. We can see a peak for $\Delta \mathrm{L}$ ong $<20^{\circ}$, and for $\Delta t$ between 0 and -4 hours, which seems to indicate that it is a precursor emission. However, the lower panel shows that the average value in this cell is related to only 14 events, which is not very significant. In general, the number of events in each cell is small, and thus the statistics with $\Delta t$ are not meaningful because of too few events. This is not the case for the ( $\Delta$ Long, $\Delta$ Inv.lat) plots. If we return to the lower panel of Plate 1, the situation is different because the polar orbit of the satellite favors the number of events in each cell: as the satellite stays at nearly the same longitude when the orbit is between $45^{\circ}$ and $+45^{\circ}$ in latitude, the same event can be seen at different invariant latitudes.

\section{Conclusions}

Data from the low-orbiting satellite AUREOL 3 were used to search for a correlation between seismic activity and increases in wave emissions. Although a correlation between two phenomena does not necessarily relate to a causative link, it is shown that wave intensity is enhanced at frequencies lower than $800 \mathrm{~Hz}$ close to epicenters. There is an extension of the increase in latitude, but not in longitude, which agrees with the observations already reported by Parrot and Lefeuvre [1985], and Serebryakova et al. [1992].

The discrepancy with the paper of Henderson et al. [1993] could be explained by differences in the statistical methods as well as by the electromagnetic components that were used. Henderson et al. [1993] only considered electric measurements, but we have shown that such signals are disturbed by natural electrostatic noise.

However, our data sets only contain a limited number of events because the telemetry of the satellite was not continuously recording around the Earth. The data sets are thus biased and do not allow a complete statistical study. For this reason, it is impossible to determine if the wave growth seen close to epicenters is due to a precursor emission, or to the effect of acoustic-gravity waves after the shock. Moreover, a larger number of events would also permit to take into account other important parameters, such as the geomagnetic activity, the day/night effect, and the depth, magnitude, and location of the earthquakes.

Acknowledgments. The AUREOL 3 program was undertaken jointly by the Centre National d'Etudes Spatiales (CNES) in France, the Space Research Institute (IKI) and INTERCOSMOS in Russia. The author thanks J. J. Berthelier, principal investigator of the VLF experiment, for use of the data. This work was financially supported by CNES during the definition of the project DEMETER (Detection of Electro-Magnetic Emissions Tranamitted from Earthquake Regions). The many participants in this project are thanked for the helpful discussions the author had with them.

The editor thanks A. B. Draganov and A. C. Fraser-Smith for their assistance in evaluating this paper.

\section{References}

Berthelier, J.J., et al., Measurements of the VLF electric and magnetic components of waves and DC electric field on board the AUREOL 3 spacecraft: The TBF-ONCH experiment, Ann. Geophys., 38, 643-667, 1982.

Chmyrev, V.M., N.V. Isaev, S.V. Bilichenko, and G. Stanev, Observation by space-borne detectors of electric fields and hydromagnetic waves in the ionosphere over an earthquake centre, Phys. Earth Planet. Inter., 57, 110, 1989.

Cress, G.O., B.T. Brady, and G.A. Rowell, Sources of electromagnetic radiation from fracture of rock samples in the laboratory, Geophys. Res. Lett., 14, 331, 1987.

Dea, J.Y., P.M. Hansen, and W.M. Boerner, Long-term ELF background noise measurements, the existence of window regions, and applications to earthquake precursor emission studies, Phys. Earth Planet. Inter., 77, 109, 1993.

Draganov, A.B., U.S. Inan, and Yu.N. Taranenko, ULF magnetic signatures at the earth surface due to ground water flow: A possible precursor to earthquakes, Geophys. Res. Lett., 18, 1127, 1991.

Enomoto, $Y$., and $\mathrm{H}$. Hashimoto, Emission of charged particles from indentation fracture of rocks, Nature, 346, 641, 1990.

Fraser-Smith, A.C., A. Bernardi, P.R. McGill, M.M. Bowen, M.E. Ladd, R.A. Helliwell, and O.G. Villard Jr, Low-frequency magnetic field measurements near the epicenter of the $\mathrm{M}_{\mathrm{g}} 7.1$ Loma Prieta earthquake, Geophys. Res. Lett., I7, 1465, 1990.

Fujinawa, Y., and K. Takahashi, Emission of electromagnetic radiation preceding the Ito seismic swarm of 1989 , Nature, 347, 376, 1990.

Gokhberg, M.B., V.A. Morgunov, T. Yoshino, and I. Tomizawe, Experimental measurement of electromagnetic emissions possibly related to earthquakes in Japan, J. Geophys. Res., 87, 7824, 1982.

Gokhberg, M.B., V.A. Pilipenko, and O.A. Pokhotelov, Seismic precursors in the ionosphere, Izv. Russ. Acad. Sci. Phys. Solid Earth, Engl. Transl., 19, 762, 1983.

Gokhberg, M.B., N.I. Gershenzon, I.L. Gufel'd, A.V. Kustov, V.A. Liperovskiy, and S.S. Khusameddimov, Possible effects of the action of electric fields of seismic origin on the ionosphere, Geomagn. Aeron., Engl. Transl., 24, 183, 1984.

Hayakawa, M., T. Yoshino, and V.A. Morgounov, On the possible influence of seismic activity on the propagation of magnetospheric whistlers at low latitudes, Phys. Earth Planet. Inter., 77, 97, 1993.

Henderson, T.R., V.S. Sonwalkar, R.A. Helliwell, U.S. Inan, and A.C. Fraser-Smith, A search for ELF/VLF emissions induced by earthquakes as observed in the ionosphere by the DE 2 satellite, J. Geophys. Res., 98, 9503, 1993.

Holtet, J.A., N.C. Maynard, and J.P. Heppner, Variational electric field at low latitudes and their relation to spread- $F$ and plasma irregularities, J. Atmos. Terr. Phys., 39, 247, 1977. 
Kelley, M.C., and F.S. Mozer, A satellite survey of vector electric fields in the ionosphere at frequencies of 10 to $500 \mathrm{Hertz}, 3$, Low-frequency equatorial emissions and their relationship to ionospheric turbulence, J. Geophys. Res., 77, 4183, 1972.

Kelley, M.C., B.T. Tsurutani, and F.S. Mozer, Properties of ELF electromagnetic waves in and above the Earth's ionosphere deduced from plasma wave experiments on the OV1-17 and OGO 6 satellites, J. Geophys. Res., 80, 4603, 1975.

Koons, H.C., and J.L. Roeder, ELF measurements at the Emerson fault following the Landers, California earthquake, Aerospace Rep. ATR-93(8351)-1, The Aerospace Corp., El Segundo, Calif., 1993.

Larkina, V.I., A.V. Nalivayko, N.I. Gershenzon, M.B. Gokhberg, V.A. Liperovskiy, and S.L. Shalimov, Observations of VLF emissions related with seismic activity, on the Intercosmos 19 satellite, Geomagn. Aeron., Engl. Transl., 23, 684, 1983.

Larkina, V.I., V.V. Migulin, O.A. Molchanov, I.P. Kharkov, A.S. Inchin, and V.B. Schvetcova, Some statistical results on very low frequency radiowave emissions in the upper ionosphere over earthquake zones, Phys. Earth Planet. Inter., 57, 100, 1989.

Lockner, D.A., M.J.S. Johnston, and J.D. Byerlee, A mechanism to explain the generation of earthquake lights, Nature, 302, 28, 1983.

Mikhaylova, G.A., A.M. Golyavin, and Yu.M. Mikhaylov, Dynamic spectra of VLF-radiation in the outer ionosphere associated with the Iranian earthquake of June 21, 1990 (Intercosmos 24 satellite), Geomagn. Aeron., Engl. Transl., 31, 647, 1991.

Molchanov, O.A., Transmission of electromagnetic fields from seismic sources to the upper ionosphere, Geomagn. Aeron., Engl. Transl., 31, 80, 1991.

Molchanov, O.A., O.A. Mazhaeva, A.N. Goliavin, and M. Hayakawa, Observation by the Intercosmos-24 satellite of ELF-VLF electromagnetic emissions associated with earthquakes, Ann. Geophys., 11, 431, 1993.

Oike, K., and T. Ogawa, Electromagnetic radiations from shallow earthquakes observed in the LF range, J. Geomagn. Geoelectr. 38, $1031,1986$.

Parrot, M., World map of ELF/VLF emissions as observed by a low-orbiting satellite, Ann. Geophys., 8, 135, 1990.

Parrot, M., and F. Lefeuvre, Correlation between GEOS VLF emissions and earthquakes, Ann. Geophys., 3, 737, 1985.

Parrot, M., and M.M. Mogilevsky, VLF emissions associated with earthquakes and observed in the ionosphere and the magnetosphere, Phys. Earth Planet. Inter., 57, 86, 1989.

Parrot, M., J. Achache, J.J. Berthelier, E. Blanc, A. Deschamps, F. Lefeuvre, M. Menvielle, J.L. Plantet, P. Tarits, and J.P. Villain, High-frequency seismo-electromagnetic effects, $P$ hys. Earth Planet. Inter., 77, 65, 1993.

Scarabucci, R.R., Satellite observations of equatorial phenomena and defocusing of VLF electromagnetic waves, J. Geophys. Res., 75, 69, 1970.

Serebryakova, O.N., S.V. Bilichenko, V.M. Chmyrev, M. Parrot, J.L. Rauch, F. Lefeuvre, and O.A. Pokhotelov, Electromagnetic ELF radiation from earthquake regions as observed by lowaltitude satellites, Geophys. Res. Lett., 19, 91, 1992.

U.S. Department of the Interior / Geological Survey, Preliminary determination of epicenters, Monthly listing, Natl. Earthquake Inf. Serv., Denver, 1981-1983.

Warwick, J.W., C. Stoker, and T.R. Meyer, Radio emission associated with rock fracture: Possible application to the great Chilean earthquake of May 22, 1960, J. Geophys. Res., 87, $2851,1982$.

M. Parrot, Laboratoire de Physique et Chimie de l'Environnement, CNRS, 3A, Avenue de la Recherche Scientifique, 45071 Orléans cedex 02, France. (e-mail: mparrot@cnrs-orleans.fr)

(Received November 22, 1993; revised July 7, 1994; accepted August 11, 1994.) 